\title{
DETECCIÓN DE TETRACICLINAS EN NUEVE LOTES DE ALIMENTOS PARA CERDOS, TILAPIAS Y POLLOS PRODUCIDOS EN COSTA RICA: INCUMPLIMIENTO DE NORMATIVAS Y DISCONFORMIDADES CON EL ETIQUETADO OFICIAL DE GARANTÍA
}

\author{
Karla Gutiérrez,, Margarita Alfaro**, Fabio Granados**, \\ Jorge Sánchez ${ }^{* *}$, Fernando García*, César Rodríguez ${ }^{1 / *}$
}

Palabras clave: Tetraciclinas, nutrición animal, cerdos, pollos de engorde, tilapia, etiquetado.

Keywords: Tetracyclines, animal feed, swine, tilapia, broiler chicken, labeling.

Recibido: 04/06/10

\section{RESUMEN}

Sobre criterios de riesgos ambientales y sanitarios asociados al uso de antibióticos en la producción animal, se utilizó un método microbiológico y un método analítico por HPLC para cuantificar tetraciclinas y determinar su identidad, respectivamente, en lotes de alimento para cerdos $(n=4)$, tilapia $(n=3)$ y pollos de engorde $(n=2)$ producidos en Costa Rica. Tres alimentos para consumo de cerdos y 2 alimentos para consumo de tilapias dieron resultados positivos por ambas técnicas. Los alimentos para cerdos positivos contenían clortetraciclina en concentraciones superiores a las permitidas por los reglamentos vigentes en el país y los alimentos de tilapia positivos contenían oxitetraciclina en concentraciones iguales o mayores a las detectadas en los alimentos para cerdos. Los alimentos para pollos, por el contrario, no contenían tetraciclina, ni oxitetraciclina ni clotetraciclina. Se hallaron incongruencias entre las concentraciones de tetraciclinas detectadas y las especificadas en la etiqueta de 4 muestras. Estos resultados justifican una mayor vigilancia por parte de los productores, consumidores y autoridades sanitarias costarricenses.

1 Autor para correspondencia. Correo electrónico: cesar.rodriguezsanchez@ucr.ac.cr

Centro de Investigación en Enfermedades Tropicales (CIET), Universidad de Costa Rica.
Aceptado: 07/09/10

\begin{abstract}
Microbiological detection of tetracyclines in 9 lots of animal feed produced in Costa Rica: Infringement of norms and incongruences with the official labeling. Considering the environmental and sanitary risks associated with the use of antibiotics in animal production, we used a microbiological method and an analytical method by HPLC to evaluate the presence of tetracyclines in 9 lots of animal feed for swine $(\mathrm{n}=4)$, tilapia fish $(\mathrm{n}=3)$ and broiler chicken $(\mathrm{n}=2)$ produced in Costa Rica. Up to $75 \%$ and $67 \%$ of the swine and tilapia feed samples analyzed gave positives results using both techniques, respectively. By contrast, the broiler chicken feed samples did not contain any of the three tetracyclines included in the tests. All positive swine feed samples contained chlortetracycline in concentrations higher than those allowed by the Costa Rican regulation. Positive tilapia feed samples contained oxitetracycline in concentrations equal or greater than those detected in swine feed. In addition, four samples exhibited inconsistencies between the concentrations of tetracyclines detected and the concentrations specified in their labels. These results justify a stronger monitoring by the producers, consumers and national sanitary authorities.
\end{abstract}

\footnotetext{
** Centro de Investigación en Nutrición Animal (CINA), Universidad de Costa Rica.
} 


\section{INTRODUCCIÓN}

Debido a su fácil absorción, alta toxicidad selectiva y bajo costo, las tetraciclinas se utilizan como agentes terapéuticos, profilácticos o estimulantes de crecimiento en ganado de engorde, aves de corral, cerdos, cabras, caballos, conejos, camarones, tilapias, y salmones, entre otros (Michalova et al. 2004, Scott y Fedorka-Cary 2002, Schwarz et al. 2001).

Solamente en el 2008, Costa Rica importó $27081 \mathrm{~kg}$ de productos agropecuarios que contienen oxitetraciclina como principal ingrediente activo (Servicio Fitosanitario del Estado 2009). Sin embargo, este tipo de productos se utilizan inadecuadamente debido a la falta de programas que concienticen al productor sobre los efectos nocivos que el uso irracional de los mismos tiene sobre el ecosistema de la unidad de producción pecuaria y la falta de programas de monitoreo y control por parte de las autoridades gubernamentales.

Esta situación desatendida tiene graves implicaciones ambientales y sanitarias ya que los antibióticos administrados a los animales terrestres o acuáticos en el alimento, las bacterias resistentes y los genes de resistencia asociados pueden persistir en los suelos o sedimentos (Chander et al. 2005, Thorsten et al. 2003, Wung-Jones 2008), alcanzar fuentes de aguas subterráneas (Witte 2000), diseminarse a otros sitos a través de fertilizantes preparados con excretas de animales tratados o aguas de riego contaminadas (Jurgensen y Halling-Sorensen 2000, Ghosh y LaPara 2007), o entrar en contacto con los consumidores si se absorben o adsorben a cultivos o cárnicos (Gaunf y Piddock 1996, Katz y Ward 2005). Por otro lado, el mal uso y abuso de los antibióticos en la agricultura costarricense puede tener un impacto negativo en la economía del país, ya que los productos pueden llegar a ser rechazados por los consumidores locales y los importadores debido a que no cumplen con los estándares de calidad esperados.

Debido a que a nivel nacional no se controla de forma rutinaria la concentración de tetraciclinas en alimentos para consumo animal, el objetivo de la presente investigación fue aplicar un método microbiológico para la cuantificación de oxitetraciclina a 9 muestras de alimento para cerdo $(n=4)$, tilapia $(n=3)$ y pollo $(n=2)$ producidas en Costa Rica. Los resultados fueron corroborados cualitativamente mediante un método por HPLC y comparados con la reglamentación vigente y con las etiquetas con que los alimentos fueron inscritos en el Registro de Control de Calidad de la Dirección de Alimentos para Animales (Servicio Nacional de Salud Animal, Ministerio de Agricultura y Ganadería de Costa Rica).

\section{MATERIALES Y MÉTODOS}

\section{Recolección de muestras de alimentos de consumo animal}

Se colectaron muestras de al menos un $\mathrm{kg}$ de alimento en 5 empresas costarricenses productoras de alimento para cerdos, pollos o tilapias durante enero y agosto de 2009 (Cuadro 1). No se analizaron más muestras debido a la negativa de otros productores para colaborar voluntariamente en el estudio o por la no disponibilidad de lotes alternativos en las empresas visitadas. Todas las muestras fueron almacenadas por no más de un mes en un lugar seco y oscuro para evitar la degradación de los analitos antes de ser analizadas.

\section{Detección microbiológica de tetraciclinas}

La detección microbiológica de tetraciclinas se basó en 2 modificaciones del método 968.50 de la Asociación de Comunidades Analíticas (AOAC International 2000). Este procedimiento fue originalmente diseñado para detectar oxitetraciclina a través de la inhibición del crecimiento en plato de una cepa sensible de Bacillus cereus (ATCC 11 778), sin embargo, no discrimina entre este analito y otras tetraciclinas.

La primera modificación consistió en obtener las esporas de Bacillus cereus ATCC 11 778 en platos de agar nutritivo suplementado con $50 \mathrm{mg} . \mathrm{l}^{-1} \mathrm{MnSO}_{4} \cdot \mathrm{H}_{2} \mathrm{O}, 100 \mathrm{mg} \cdot \mathrm{l}^{-1} \mathrm{CaCl}_{2} \cdot 2 \mathrm{H}_{2} \mathrm{O}$ y 
Cuadro 1. Cuantificación microbiológica e identificación por HPLC de tetraciclinas en alimentos para consumo animal producidos en Costa Rica.

\begin{tabular}{|c|c|c|c|c|c|c|}
\hline Empresa & Muestra & Tipo de alimento & Lote & $\begin{array}{l}\text { Antibiótico indicado en } \\
\text { la etiqueta }\left(\mathrm{mg} \cdot \mathrm{kg}^{-1}\right)\end{array}$ & 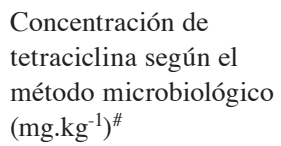 & $\begin{array}{l}\text { Tetraciclina } \\
\text { identificada por } \\
\text { HPLC }\end{array}$ \\
\hline A & A1 & Tilapia (engorde) & 17942 & $\begin{array}{l}\text { Oxitetraciclina } \\
\text { (no indica) }\end{array}$ & $376 \pm 0,05$ & Oxitetraciclina \\
\hline A & A5 & Tilapia (desarrollo) & 17985 & Ninguno & 0 & Ninguno \\
\hline $\mathrm{B}$ & B2 & Tilapia (adulto) & $\mathrm{ND}^{\&}$ & Ninguno & $261 \pm 0,05$ & Oxitetraciclina \\
\hline $\mathrm{C}$ & $\mathrm{C} 3$ & Cerdo (inicio) & ND & Clortetraciclina (2000) & $260 \pm 0,05$ & Clortetraciclina \\
\hline $\mathrm{C}$ & $\mathrm{C} 4$ & Cerdo (inicio) & ND & Ninguno & $87 \pm 0,05$ & Clortetraciclina \\
\hline $\mathrm{C}$ & $\mathrm{C} 7$ & Cerdo (inicio) & ND & $\begin{array}{l}\text { Clortetraciclina } \\
(50)\end{array}$ & $84 \pm 0,05$ & Clortetraciclina \\
\hline $\mathrm{D}$ & D6 & Cerdo (inicio) & 4514 & $\begin{array}{l}\text { Bacitracina } \\
\text { metilendisalicilato } \\
\text { (22); Fenbendazol (4); } \\
\text { Feniclor (62) }\end{array}$ & 0 & Ninguno \\
\hline $\mathrm{E}$ & E8 & Pollo (crecimiento) & ND & Ninguno & 0 & Ninguno \\
\hline $\mathrm{E}$ & E9 & Pollo (crecimiento) & ND & Ninguno & 0 & Ninguno \\
\hline
\end{tabular}

\&ND: Información no disponible.

\# $\quad$ Esta concentración se determina usando curvas de calibración que describen la relación lineal entre el tamaño de los halos de inhibición del crecimiento de Bacillus cereus ATCC 11778 y la concentración de un patrón de oxitetraciclina o tetraciclina presente en un plato de agar Agar Nutritivo suplementado con $50 \mathrm{mg} . \mathrm{l}^{-1} \mathrm{MnSO}_{4} \cdot \mathrm{H}_{2} \mathrm{O}, 100 \mathrm{mg} .1^{-1} \mathrm{CaCl}_{2} \cdot 2$ $\mathrm{H}_{2} \mathrm{O}$ y $500 \mathrm{mg} \cdot \mathrm{l}^{-1}$ de $\mathrm{MgSO}_{4} \cdot 7 \mathrm{H}_{2} \mathrm{O}$.

$500 \mathrm{mg} . \mathrm{l}^{-1}$ de $\mathrm{MgSO}_{4} \cdot 7 \mathrm{H}_{2} \mathrm{O}$, en lugar de platos de agar antibiótico $\mathrm{N}^{\circ} .1$ debido a la pobre esporulación de la cepa en estos últimos platos. Por otro lado, y para incrementar el ámbito de concentraciones de tetraciclinas detectables, calculamos curvas de calibración con 9 concentraciones de oxitetraciclina y tetraciclina $(0,1 ; 0,15 ; 0,2 ; 0,3$; 0,$4 ; 0,5 ; 0,6 ; 0,8$ y 1,0 mg. $\mathrm{kg}^{-1}$ ) en lugar de las 5 concentraciones de oxitetraciclina recomendadas por el método $\left(0,1 ; 0,15 ; 0,2 ; 0,3\right.$ y $\left.0,4 \mathrm{mg}_{\mathrm{kg}}{ }^{-1}\right)$. Estas curvas de calibración relacionan el halo de inhibición del crecimiento de Bacillus cereus ATCC 11778 (variable dependiente, eje Y) con la concentración de patrón de oxitetraciclina o tetraciclina añadida in vitro (variable independiente, eje X). Al mismo tiempo, se realizaron experimentos de recuperación por medio del método modificado a una muestra de alimento libre de tetraciclinas a la que se le añadieron concentraciones conocidas de oxitetraciclina o tetraciclina.

La preparación de las muestras, la extracción de las tetraciclinas con metanol a partir de los alimentos, la inoculación e incubación de los platos con el biosensor, y la lectura de los halos de inhibición se realizó sin modificaciones. La concentración de tetraciclinas en las muestras se determinó al interpolar el tamaño del halo de inhibición causado por los extractos de los alimentos en las curvas de calibración arriba mencionadas (preparadas con estándares 
de concentración conocida). Los resultados obtenidos representan el promedio de 6 repeticiones, para lo que se utilizó agua destilada estéril como control negativo.

\section{Identificación de tetraciclinas por HPLC}

Para corroborar que la inhibición del crecimiento de Bacillus cereus ATCC 11778 se debiera a la presencia de oxitetraciclina, tetraciclina, o clortetraciclina, los alimentos fueron analizados de forma cualitativa mediante HPLC. Para esta cromatografía en fase reversa se utilizó una columna SB-C8 (150 x 4,6 mm, $5 \mu \mathrm{m})$ y una mezcla 45:50:5 de acetonitrilo, agua acidificada con $0,1 \mathrm{mmol}^{\mathrm{m}} \mathrm{m}^{-1}$ ácido trifluoroacético y metanol como fase móvil. La separación fue isocrática, con un flujo de fase móvil ajustado a 0,5 ml.min ${ }^{-1}$, una temperatura de columna de $30^{\circ} \mathrm{C}$, e inyectado $20 \mu \mathrm{L}$ de las muestras. Los analitos se visualizaron con un detector ultravioleta a 355 $\mathrm{nm}$ y un detector de fluorescencia $\left(\lambda_{\text {emisión }}=390\right.$ $\mathrm{nm} ; \lambda_{\text {excitación }=} 493 \mathrm{~nm}$ ) posterior a una derivatización post-columna con $0,1 \mathrm{mmol} \cdot \mathrm{ml}^{-1} \mathrm{AlCl}_{3} \cdot 6 \mathrm{H}_{2} 0$ $(\mathrm{pH}=3,4)$ a $60^{\circ} \mathrm{C}$. Como controles se utilizaron oxitetraciclina dihidratada (SigmaUltra, lote 112H01231), clortetraciclina hidrocloruro (Sigma, lote 127F-0609) y tetraciclina hidrocloruro (BioChemika, Fluka, lote 431039/1 60203038).

Este procedimiento no se usó para determinar la concentración de oxitetraciclina, tetraciclina, o clortetraciclina en los alimentos porque no permite determinar la fracción biodisponible responsable de sus efectos biológicos y además porque no permite detectar sustancias inhibitorias del crecimiento de Bacillus cereus ATCC 11778 que no fluorezcan ni se derivaticen con $\mathrm{AlCl}_{3} \cdot 6 \mathrm{H}_{2} 0$.

\section{Normas para la interpretación de los resultados}

La normativa que regula el uso de antibióticos en alimentos para animales en Costa Rica se ampara en los capítulos VII "De las indicaciones para el uso de medicamentos aditivos y nitrógeno no proteico", y IX “Medicamentos y aditivos" del reglamento a la Ley de la República 6683, en su decreto $\mathrm{N}^{\circ} .16$ 899-Ministerio de Agricultura y Ganadería (MAG).

Las referencias que utiliza la Dirección de Alimentos para Animales del Servicio Nacional de Salud Animal del Ministerio de Agricultura y Ganadería para aprobar medicamentos o cualquier aditivo son los reglamentos de la Administración de Alimentos y Drogas del Departamento de Salud y Servicios Humanos de EE. UU (FDA). En este sentido, la FDA establece que la oxitetraciclina como promotor de crecimiento para cerdos y pollos debe añadirse a los alimentos en concentraciones entre 11 y $55 \mathrm{mg} \cdot \mathrm{kg}^{-1}$. Este mismo antibiótico debe añadirse a una concentración de $22 \mathrm{mg} \cdot \mathrm{kg}^{-1}$ y $110-550 \mathrm{mg} \cdot \mathrm{kg}^{-1}$ para el tratamiento de enfermedades en cerdos y pollos, respectivamente. En el caso de las tilapias, solamente se permite el uso de la oxitetraciclina con fines terapéuticos en concentraciones menores o iguales a 2,5-3,5 g/ 45,5 kg de pez.día ${ }^{-1}$. Este dato se obtuvo con la extrapolación de los valores que se estipulan para pez gato y salmonoides debido a que la FDA no considera el caso específico para tilapia. La FDA recomienda que la clortetraciclina se añada en la industria porcina en concentraciones entre $11-55 \mathrm{mg} \cdot \mathrm{kg}^{-1}$ como promotor de crecimiento y entre $55-440 \mathrm{mg} . \mathrm{kg}^{-1}$ como agente terapéutico.

\section{RESULTADOS}

Las 2 modificaciones realizadas al método 968.50 permiten detectar, con una correlación lineal de $r^{2}=0,95$ y una reproducibilidad con coeficientes de variación alrededor del $0,1 \%$, entre $0,1 \mu \mathrm{g} \mathrm{m} . \mathrm{l}^{-1}$ y $1,0 \mu \mathrm{g} \mathrm{m} . \mathrm{l}^{-1}$ de tetraciclina y de oxitetraciclina. Los porcentajes de recuperación para tetraciclina y oxitetraciclina fueron del $91 \%$ y $82 \%$, respectivamente. La especificidad del método se corroboró al observar una disminución cercana al $35 \%$ en los diámetros de inhibición cuando los extractos metanólicos fueron diluidos con $0,5 \mathrm{M} \mathrm{CaCl}_{2}$ en lugar de agua destilada estéril (datos no mostrados). 
De acuerdo al método microbiológico, 3 alimentos para consumo de cerdos (C3, C4 y C7) y 2 alimentos para consumo de tilapias (A1 y B2) contenían tetraciclinas (Cuadro 1). El método por HPLC, por su parte, demostró que las muestras que dieron resultados positivos en el análisis microbiológico contenían oxitetraciclina o clortetraciclina (Cuadro 1).

Los 3 alimentos para consumo de cerdos con resultados positivos $(\mathrm{C} 3, \mathrm{C} 4, \mathrm{C} 7)$ contenían clortetraciclinas en concentraciones superiores a las permitidas en la legislación. Por su parte, los 2 alimentos para tilapia positivos (A1 y B2) contenían oxitetraciclina en concentraciones iguales o superiores a las detectadas en los alimentos para cerdos.

Solamente 3 de los 9 alimentos analizados tenían la información completa en las etiquetas (Cuadro 1). La concentración de tetraciclinas detectada en el alimento para tilapia B2 y en los alimentos para cerdos $\mathrm{C} 4$ y C7 fue mayor que la reportada por el productor (Cuadro 1). Dos de estos alimentos no debían contener tetraciclinas (B2, C4). La situación inversa también fue observada, ya que el alimento para cerdos C3 mostró una concentración de clortetraciclina menor a la reportada en la etiqueta de garantía.

\section{DISCUSIÓN Y CONCLUSIONES}

Los porcentajes de recuperación observados en este estudio son congruentes con los reportados para este tipo de matrices (Wang et al. 2008). Por otro lado, la disminución de la actividad de las tetraciclinas en presencia de calcio se debe a un fenómeno de quelación y precipitación por parte de cationes divalentes, lo que impide el transporte del antibiótico a través de membranas celulares (Palm et al. 2008).

Los alimentos para consumo animal son comúnmente suplementados con calcio y cationes divalentes que podrían disminuir la concentración biodisponible de las tetraciclinas. Por tanto, los resultados aquí expuestos probablemente subestiman la concentración total de tetraciclinas presentes en los alimentos analizados. De todas formas, y a pesar del número reducido de muestras analizadas, los resultados indican que algunos alimentos para consumo de cerdo y tilapia producidos en Costa Rica pueden contener clortetraciclina (cerdos) u oxitetraciclina (tilapia) en concentraciones no permitidas y/o incongruentes con las especificadas en las etiquetas.

Desde una perspectiva ambiental y al considerar que la crianza de cerdos así como de tilapias son actividades que generan toneladas de excrementos con altas concentraciones de tetraciclinas y metabolitos activos día a día (Hansen et al. 2002, Chen et al. 2010), se anticipa una alta prevalencia de bacterias resistentes y de genes de resistencia a tetraciclinas en agroecosistemas costarricenses donde se utilicen fertilizantes preparados con cerdaza o aguas de riego que hayan tenido exposición directa o indirecta a porquerizas y fincas de tilapia (Knapp et al. 2010, Zhang et al.2009, Bustamante et al. 2003). Esta situación es preocupante, ya que compromete la utilidad de estas drogas en las actividades agropecuarias (Vidaver 2002) y en la medicina humana y veterinaria (McEwen y Fedorka-Cray 2002, Witte 1998, Swartz 2002).

Algunos alimentos para consumo animal producidos en Costa Rica, así como productos agrícolas obtenidos en fincas donde los animales reciben estos alimentos, podrían ser susceptibles de sanciones o cierre de mercados debido a la presencia de bacterias resistentes, genes de resistencia y/o antibióticos en concentraciones no permitidas o incongruentes con el etiquetado. Un ejemplo sería el caso de Europa, en donde el uso de antibióticos está prohibido en la nutrición animal. Esta situación ha sido abordada en los últimos años por autoridades sanitarias en países desarrollados (FAO/OIE/WHO 2006, WHO 1997) y se ha discutido ampliamente en la redacción del último Codex Alimentarius de la Organización de las Naciones Unidas para la Agricultura y la Alimentación (FAO 2009).

Las diferencias entre las concentraciones indicadas en las etiquetas y las encontradas en los productos podrían deberse a fallas en el proceso de limpiado de los tambores de mezcla o al uso de 
antibióticos de dudosa calidad, entre otros. Este último aspecto es crítico debido a que la calidad del antibiótico que se utiliza como materia prima tiene un impacto directo en su biodisponibilidad y actividad biológica (Barr 1969).

Se recomienda a los consumidores de alimentos para animales producidos en Costa Rica informarse acerca de los procesos de manufactura utilizados por las empresas nacionales, exigir un apropiado etiquetado y evaluar constantemente la composición y homogeneidad de los lotes de alimento que compran. Al mismo tiempo, instamos a los productores a vigilar la composición de sus alimentos de forma estricta y a seguir las buenas prácticas de manufactura recomendadas para este tipo de procesos y a las autoridades sanitarias costarricenses a instaurar programas de monitoreo y regulación del uso de antibióticos en las actividades agropecuarias.

\section{AGRADECIMIENTOS}

Esta investigación fue financiada por el Consejo Nacional de Rectores (CONARE) a través del Fondo de Estímulo para la Educación Superior (FEES) y forma parte del proyecto "Detección de antibióticos y resistencia antimicrobiana en la agricultura, acuacultura y nutrición animal desarrollada en el distrito de riego ArenalTempisque y parte interna del Golfo de Nicoya" (803-A8-164). Los autores expresan su gratitud a las empresas que facilitaron los alimentos analizados.

\section{LITERATURA CITADA}

ASSOCIATION OF OFFICIAL ANALYTICAL CHEMIST (AOAC). 2000. Official Method of Analysis. Chapter 5: AOAC Official Method 968.50. Oxytetracycline in Feed. Vol II. 56 p.

BARR WH. 1969. Factors involved in the assessment of systemic or biological availability of drug products. Drug Inf. Bull. 3:2745.

BUSTAMANTE W., ALPÍZAR A., HERNÁNDEZ S., PACHECO A., VARGAS N., HERRERA M., HERRERA A., CABALLERO M., GARCÍA F.
2003. Predominance of vanA Genotype among Vancomycin-Resistant Enterococcus Isolates from Poultry and Swine in Costa Rica. Appl. Environ. Microbiol. 69(12):7414-7419.

CHANDER Y., KUMAR K., GOYAL S., GUPTA S. 2005. Antibacterial Activity of Soil-Bound Antibiotics. J. Environ. Qual. 34:1952-1957.

CHEN J., MICHEL F., SREEVATSAN S., MORRISON M., YU Z. 2010. Occurrence and Persistence of Erythromycin Resistance Genes (erm) and Tetracycline Resistance Genes (tet) in Waste Treatment Systems on Swine Farms. Microb. Ecol. DOI 10.1007/s00248-010-9634-9635.

MAG (Ministerio de Agricultura y Ganadería). 2010. Servicio Fitosanitario del Estado. Costa Rica. 17/01/10. http:// www.protecnet.go.cr/consultasweb_sicoin.

FAO (Food and Agriculture Organization). 2009. Documento CX/AMR 09/3/4.

GAUNF P., PIDDOCK L. 1996. Ciprofloxacin resistant Campylobacter spp. in humans: an epidemiological and laboratory study. J. Antimicrob. Chemother. 37:747-757.

GHOSH S., LAPARA T. 2007. The effects of subtherapeutic antibiotic use infarm animals on the proliferation and persistence of antibiotic resistance among soil bacteria. ISME J. 1:191-203.

HANSEN L., AARESTRUP F., SORENSEN S. Quantification of bioavailable chlortetracycline in pig feces using a bacterial whole-cell biosensor. 2002. Vet. Microbiol. 87:51-57.

JURGENSEN S., HALLING-SÙRENSEN B. 2000. Drugs in the environment. Chemosphere 40:691-699.

KATZ S-E., WARD P-M. 2005. Antibiotic and Hormone Residues in Foods and their Significance. In: Zdzislaw E-S y Waldemar M-D. Toxins in Food. CRC PRESS

KNAPP C., DOLFING J., EHLERT P., GRAM D. 2010. Evidence of Increasing Antibiotic Resistance Gene Abundances in Archived Soils since 1940. Environ. Sci. Technol. 44:580-587.

MCEWEN S., FEDORKA-CRAY P. 2002. Antimicrobial Use and Resistance in Animals. Clin. Infect. Dis. 34 (suppl 3):93. 
MICHALOVA E., NOVOTNA J., SCHLEGELOVA J. 2004. Tetracyclines in veterinary medicine and bacterial resistance to them. Vet. Med. 49(3):79-100.

PALM G., LEDERER T., ORTH P., SAENGER W., TAKAHASHI M., HILLN W., HINRICHS W. 2008. Specific binding of divalent metal ions to tetracycline and to the Tet repressor/tetracycline complex. J. Biol. Inorg. Chem. 13:1097-1110.

SCHWARZ S., KEHRENBERG C., WALSH T. 2001. Use of antimicrobial agents in veterinary medicine and food animal production. Int. J. Antimicrob. Agents 17:431-437.

SCOTT A., FEDORKA-CARY P. 2002. Antimicrobial Use and Resistance in Animals. Clin. Infect. Dis. 34 (suppl 3):93-104.

SWARTZ M. 2002. Human Diseases Caused by Foodborne Pathogens of Animal Origin. Clin. Infect. Dis. 34 (Suppl 3):111.

THORSTEN C., SCHNEIDER R., FÄRBER H., SKUTLAREK D., GOLDBACH H. 2003. Determination of Antibiotic Residues in Manure, Soil and Surface Waters. Acta Hydrochim. Hydrobiol. 31:1, 36-44.

VIDAVER A. 2002. Uses of Antimicrobials in Plant Agriculture. Clin. Infect. Dis. 34 (suppl 3):107.

WANG L., YANG H., ZHANG C., MO Y., LU X. 2008. Determination of oxytetracycline, tetracycline and chloramphenicol antibiotics in animal feeds using subcritical water extraction and high performance liquid chromatography. Anal Chim Acta. 619(1):54-8.

WITTE W. 1998. BIOMEDICINE: Medical Consequences of Antibiotic Use in Agriculture. Science. 27(5353):996997. DOI: $10.1126 /$ science.279.5353.996.

WITTE W. 2000. Ecological impact of antibiotic use in animals on different complex microflora: environment. Int. J. Antimicrob. Agents 14:321325 .

WORLD HEALTH ORGANIZATION. 1997. The medical impact of antimicrobial use in food animals. Report of a WHO Meeting. Berlin, Germany. WHO/EMC/ $\mathrm{ZOO} / 97.4$.

WORLD HEALTH ORGANIZATION. 2006. Antimicrobial Use in Aquaculture and Antimicrobial Resistance. Report of a Joint FAO/OIE/WHO Expert Consultation on Antimicrobial Use in Aquaculture and Antimicrobial Resistance Seoul, Republic of Korea. 5-17 p.

WING-JONES R. 2008. Residualidad de sustancias xenobióticas en el suelo empledas en la producción pecuaria. Agronomía Mesoamericana 19(1):99-114.

ZHANG W., STURM B., KNAPP C., GRAHAM D. 2009. Accumulation of Tetracycline Resistance Genes in Aquatic Biofilms Due to Periodic Waste Loadings from Swine Lagoons Systems. Environ. Sci. Technol. 43:7643-765. 